\title{
Feature Extraction from MEMS Accelerometer and Motion Tracking Measurements in Comparison with Smart Bands during Running ${ }^{\dagger}$
}

\author{
Andy Stamm ${ }^{1,2, *}$ and Ronny Hartanto ${ }^{1}$ \\ 1 Faculty of Technology \& Bionics, Rhine-Waal University of Applied Sciences, 47533 Cleve, Germany; \\ ronny.hartanto@hsrw.eu \\ 2 Griffith School of Engineering, Griffith University, Nathan 4111, Australia \\ * Correspondence: andy.stamm@gmail.com, Tel.: +49-2821-80673-607 \\ + Presented at the 12th conference of the International Sports Engineering Association, Brisbane, \\ Queensland, Australia, 26-29 March 2018.
}

Published: 14 February 2018

\begin{abstract}
Athlete monitoring is a major field of interest for professional and recreational runners as well as for coaches to improve performance and reduce injury risk. The development of inertial sensors in recent years offers the opportunity to improve the number of monitored training sessions significantly. This research used a self-developed inertial sensor in conjunction with a motion tracking system and four smart bands to record the runner's movement and extract parameters such as step numbers and frequencies. The data recorded were calibrated before it was high-pass filtered to remove gravity components from the signal. A peak detection algorithm was developed to find the number of steps, which have been further used to compare the different systems (IMU, motion capture, smart bands) and find their agreement. The results showed a very strong correlation between the IMU and the motion tracking system of $\mathrm{r}^{2}=0.998$, and an $\mathrm{r}^{2}=0.996$ between the IMU and one smart band.
\end{abstract}

Keywords: running; accelerometer; IMU; stride frequency; steps; distance; calories; smart bands

\section{Introduction}

The investigation of the performance of runners is of great interest for athletes and coaches. Technology used usually includes mostly video camera systems [1], GPS devices [2], and contact force plates [3]. Video camera systems are usually very difficult to install, to operate and very expensive. Additionally to the long setup process a time consuming digitization process has to take place before data analysis can be undertaken. Due to this complexity in conjunction with the low availability of this expensive systems, athlete tracking cannot be conducted for every training session. GPS devices allow the tracking of the exact point in the Euclidian space but they need a direct line of sight to the satellites which limits the use of these devices to outdoor training sessions. These GPS devices are also very costly which reduces its usage to professional athletes only. While major improvements in an athlete's running style can be seen by an experienced trainer without the help of technical equipment, the trainer will need the help of this kind of equipment to investigate minor improvements in the athlete's technique.

In recent years smart bands have become more available majorly due to their reduction in weight, size, and price. These devices are capable of measuring the number of steps and use these values to estimate the calories burned and distance travelled during the run. The data is usually transferred to a smartphone which allows keeping a training record for the athlete. In contrast to these smart bands, accelerometers have been used by many researchers to find gait phases, lean 
angles, accelerations, and velocities of runners [1,2,4,5]. There is no validation study, to our knowledge, which compared steps derived from smart bands with other measurement systems like IMU's and motion tracking systems.

This study aims to firstly compare the data collected from the IMU and the motion tracking system (considered gold standard in athlete monitoring) to find their agreement and secondly to compare data collected from four different smart bands with data collected from the other measurement systems to find the agreement.

\section{Methods}

\subsection{Instrumentation}

This study used a custom designed inertial measurement unit (IMU) which contains a tri-axial accelerometer with a range of $\pm 16 \mathrm{~g}$, tri-axial gyroscope (2000 degrees per second), a tri-axial magnetometer and radio capabilities as the first reference system [6]. The IMU was set to record at a sampling rate of $100 \mathrm{~Hz}$. A treadmill U.N.O. LTX6 Professional (Beny Sports Germany GmbH, Nuernberg, Germany [7]) was used as a second reference system. The four different smart bands used were: Fitbit Alta (Fitbit Inc, San Francisco, USA [8]), Samsung Gear Fit SM-R350 (Samsung Electronics GmbH, Schwalbach/Taunus, Germany [9]), Vidonn X6 (Vidonn Information Technology Co. Ltd., Shenzhen, China [10]), and Xiaomi MiBand 2 (Xiaomi Singapore Pte. Ltd., Singapore, Singapore [11]). The motion tracking system used in this research is consisting of 8 NaturalPoint OptiTrack Prime 13 cameras (Natural Point Inc., Corvallis, OR, USA [12]) which were calibrated before the experiments were started and the motion tracking software Motive [13]. The motion tracking system was set to record at a sampling rate of $200 \mathrm{~Hz}$. The IMU was attached at the center of an Optitrack Riged Body Base (Part number MCP1145) with three Optitrack M4 12.7 mm markers (Part number MKR127M4) attached to this base to form an trackable rigid body object.

\subsection{Data Collection}

Six participants (Table 1) with varying experience levels took part in this study which has been approved by a universities ethics committee. Data were collected in a laboratory. Each participant started with a low effort running trial which was basically used as a warm up run followed by another trial at low effort, two trials at medium effort and one trial at full effort each $5 \mathrm{~min}$ long. The speed of the running trial was set on the treadmill and was either $6 \mathrm{~m} / \mathrm{s}^{-8} \mathrm{~m} / \mathrm{s}^{-10} \mathrm{~m} / \mathrm{s}$ or $8 \mathrm{~m} / \mathrm{s}^{-10} \mathrm{~m} / \mathrm{s}^{-}$ $12 \mathrm{~m} / \mathrm{s}$ for low-medium-full effort respectively. The efforts were chosen by the participants in agreement with their self-judged experience and capabilities. As this study investigated the different technologies rather than the different participants it can be assumed that the speed of the different trials could be neglected.

Table 1. List of participants with their height, mass, experience, and gender.

\begin{tabular}{ccccc}
\hline Participant Number & Height $\mathbf{( c m )}$ & Mass $\mathbf{( k g )}$ & Experience & Gender \\
\hline 1 & 185 & 82 & Novice & Male \\
2 & 161 & 67 & Novice & Male \\
3 & 167 & 57 & Novice & Female \\
4 & 176 & 89 & Recreational & Male \\
5 & 171 & 62 & Recreational & Male \\
6 & 166 & 65 & Triathlete & Female \\
\hline
\end{tabular}

The IMU was attached to the sacrum of the participant by either using a belt or alternatively a velcro and special training pants. The vertical direction (aligned with the spine) was represented by $a_{x}$, the mediolateral direction by $a_{y}$, and the anterior-posterior (aligned with forward direction) by $a_{z}$. The smart bands were worn on the left lower arm in the order: FitBit, Vidonn, Samsung, and Xiaomi starting from the hand (Figure 1). 
The measured distance and calories from the treadmill as well as the data recorded by the smart bands were manually recorded while the measured data from the IMU were downloaded wirelessly into MATLAB ${ }^{\circledR}$ at the end of each running trial.

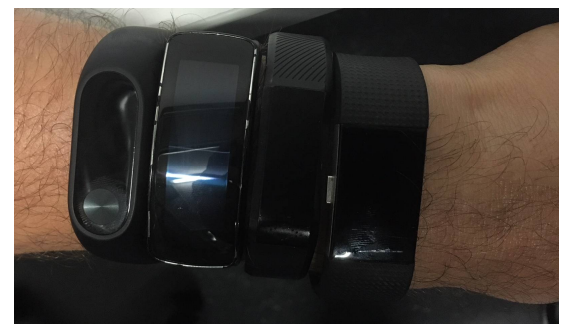

Figure 1. Smart bands and their order attached to the participants.

\subsection{Data Analysis}

The downloaded acceleration data collected by the IMU was firstly calibrated using a calibration method similar to the method described by Lai et al. [14] before it was high-pass filtered using a Hamming windowed FIR filter with a $0.5 \mathrm{~Hz}$ cut-off frequency as described by Stamm et al. [15]. This was done to remove the sensor orientation from the acceleration signal (gravity removal). Figure 2 presents a data set recorded by the IMU of one running trial (participant 1, blue) with the overlapped filtered gravity component (red).
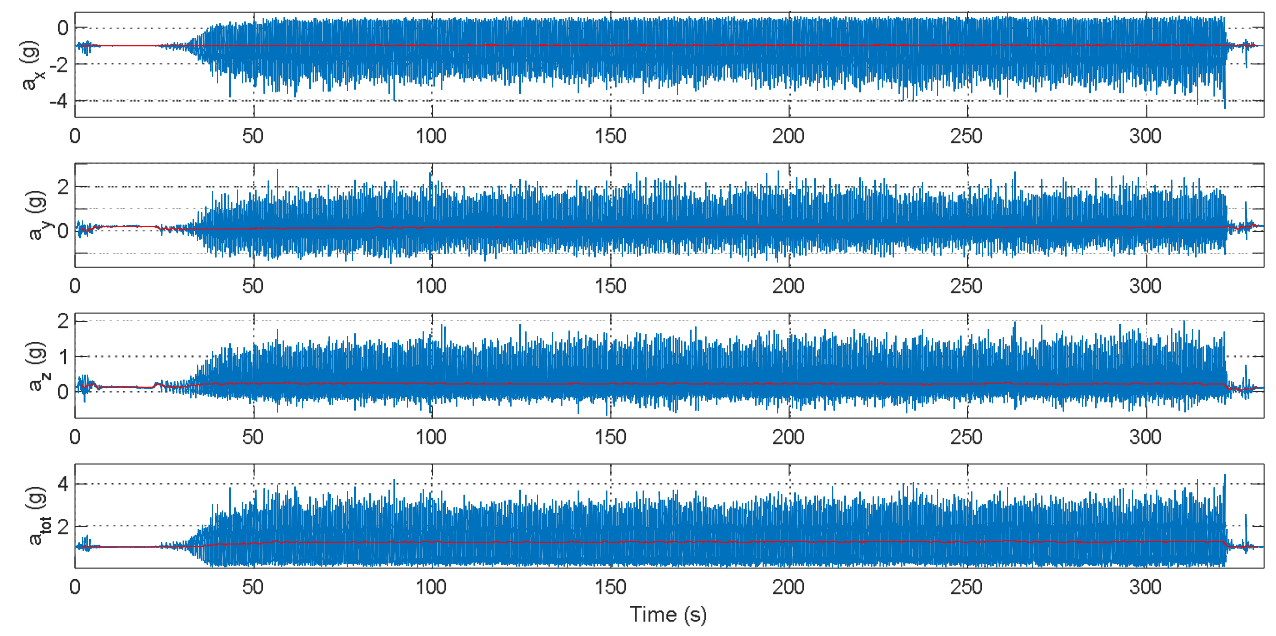

Figure 2. Data recorded by the IMU (blue) with the overlapped gravity component (red).

The vertical direction was further investigated to find the number of steps. This was achieved by applying a peak detection algorithm after the vertical direction data was filtered with a Hamming windowed FIR filter with a cut-off frequency of $5 \mathrm{~Hz}$. This way, errors in the peak detection process were reduced. Figure 3 presents the vertical direction data (blue) at the start of one low effort trial with the filtered data overlaid (red) which was used for the peak detection algorithm.

The data recorded by the motion tracking system were exported as a CSV file and imported into MATLAB $^{\circledR}$ with the built-in import tool. Figure 4a shows the $a_{y}$ channel (which was aligned with the spine) of the imported data from the Motion Tracking system where the peak detection algorithm could be applied without any further pre-processing. The implemented peak detection algorithm was used to find the number of steps for both data sets before they were stored in an array for further analysis. A linear regression analysis (coefficient of determination) comparing the number of steps (all trials) extracted from the IMU and the motion tracking system was conducted to show the alignment of both methods (Figure $4 \mathrm{~b}$ ). Additionally a Bland-Altman analysis was carried out to compare the IMU and motion tracking measurements. Further on the steps of every smart band were 
compared with the steps derived from the IMU in a similar way to find the agreement between the two methods.

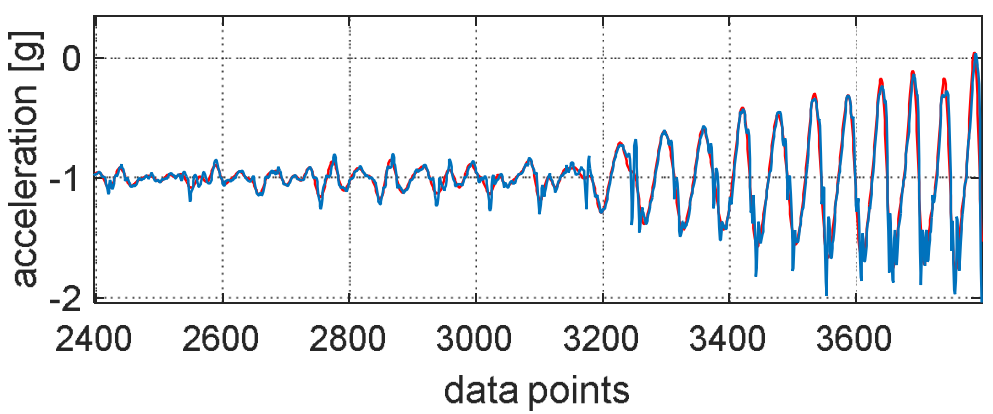

Figure 3. Vertical direction acceleration (blue) with the filtered acceleration data overlaid (red).

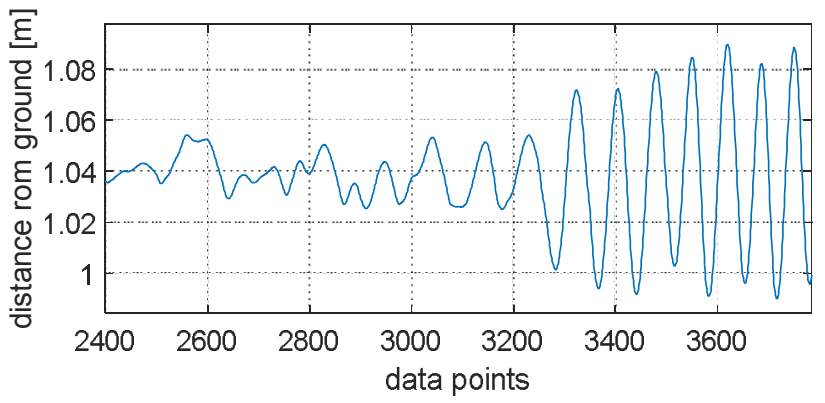

(a)

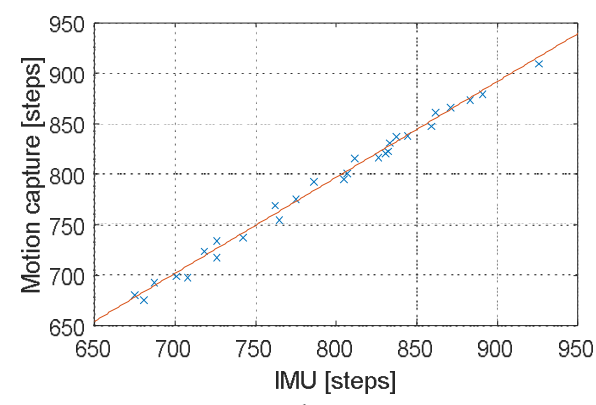

(b)

Figure 4. (a) Vertical distance recorded by the Motion Tracking system in reference to the ground; (b) Regression analysis of IMU vs. motion tracking system.

\section{Results}

One trial had to be excluded from the data analysis due to a problem with the IMU. Three further trials showed problems with the step counters of two smart bands. This left a total of 29 running trials for further analysis as the problems with the smart bands occurred only for one smart band at any time, so data analysis could be carried out for all other smart bands. Table 2 presents the steps recorded by all devices for participant 4 as one example.

Table 2. Recorded steps of all different devices for participant 4 .

\begin{tabular}{ccccccc}
\hline Runner 4 & \multicolumn{6}{c}{ Steps Recorded } \\
\hline Trial & IMU & Motion Tracking & FitBit & Samsung & Vidonn & Xiaomi \\
\hline 1 & 811 & 815 & 837 & 816 & 827 & 816 \\
2 & 833 & 831 & 830 & 837 & 828 & 840 \\
3 & 862 & 861 & 872 & 866 & 864 & 870 \\
4 & 837 & 837 & 846 & 836 & 830 & 837 \\
5 & 871 & 866 & 871 & 717 & 857 & 865 \\
\hline
\end{tabular}

Linear regression analysis of the comparison between the IMU and the motion tracking system (all conducted trials) showed that the two systems have a very strong agreement with a slope of 1.011 and an $\mathrm{r}^{2}$ of 0.998 (Figure 4b). The intercept for all calculations was set to [0, 0] as all devices were measuring the same parameter (steps).

Bland-Altman analysis was additionally used to find the agreement between these two methods (Figure 5). The result showed a bias of 3.86 with an upper limit of agreement of 17.8 and a lower limit of agreement of -9.99 with all data points inside the $95 \%$ confidence bounds. The scattering around the bias is even and follows a normal distribution with a skewness of 0.65 and a kurtosis of 2.05 (normal distribution is 3). Because of this very strong agreement, further data analysis was only 
carried out with the number of steps derived from the IMU compared with the individual smart bands. Table 3 presents the agreement of the different smart bands with the IMU.

Table 3 presents the results of the four different smart bands with their individual correlation coefficient and slope in regards to a set intercept of [0, 0]. It can be seen that the MiBand 2 from Xiaomi has the strongest agreement with the IMU with a slope of 1.006 and a correlation coefficient $\mathrm{r}^{2}$ of 0.996. Only the FitBit Alta smart band shows another good correlation with a slope of 1.003 and an $\mathrm{r}^{2}$ of 0.963 . Figure 6 presents the regression analysis of all four smart bands.

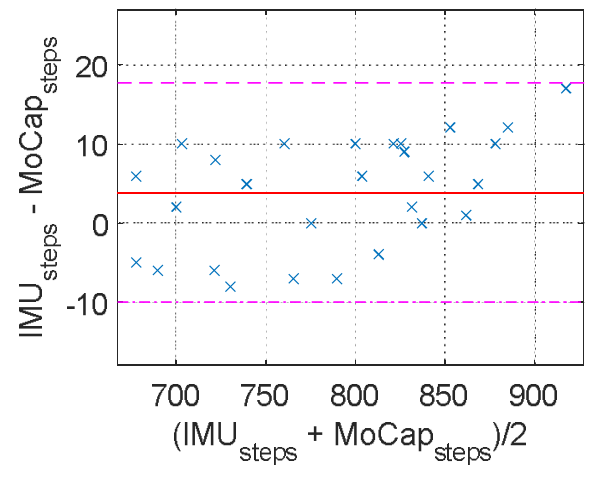

(a)

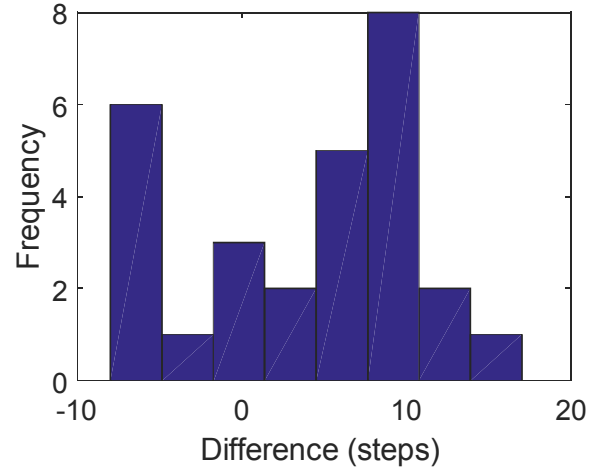

(b)

Figure 5. Statistical analysis of IMU vs. motion tracking measurements (a) Bland-Altman analysis (b) Histogram of calculated step differences.

Table 3. Results of the linear regression analysis (steps) derived from the IMU vs. the other devices.

\begin{tabular}{ccc}
\hline \multirow{2}{*}{ Device } & \multicolumn{2}{c}{ IMU } \\
\cline { 2 - 3 } & Slope & $\mathbf{r}^{\mathbf{2}}$ \\
\hline Motion tracking & 1.011 & 0.998 \\
FitBit & 1.003 & 0.963 \\
Samsung & 0.942 & 0.815 \\
Vidonn & 0.906 & 0.769 \\
Xiaomi & 1.006 & 0.996 \\
\hline
\end{tabular}

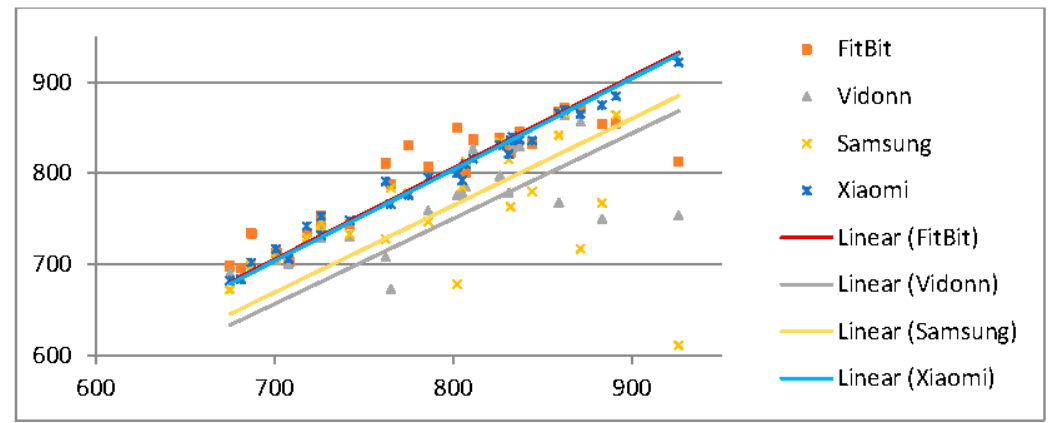

Figure 6. Regression analysis of IMU vs. smart bands.

\section{Discussion \& Conclusions}

This research investigated the number of steps during running of different participants on a treadmill utilizing a motion tracking system, a self-developed IMU attached to the sacrum of the participant and four smart bands from different brands.

The overall aim of this study was to validate the IMU sensor mounted at the lower back as being a replacement for a complex motion capture system. The comparison of the data sets derived from the IMU and the motion tracking system showed a very strong correlation $\left(\mathrm{r}^{2}=0.998\right)$ therefore it can be assumed that both systems can detect the number of steps with the same accuracy. Therefore the data recorded by the different smart bands was compared with the IMU derived data only. The 
results for the smart bands have been evaluated to be independent from the speed of the participant and trial. The validation of the four different smart bands showed that the Xiaomi MiBand 2 also had a very strong correlation of $\mathrm{r}^{2}=0.996$, the FitBit Alta had a strong correlation of $\mathrm{r}^{2}=0.963$, the Samsung Gear Fit had a good correlation of $r^{2}=0.815$, and the Vidonn X6 had a good correlation or $r^{2}=0.769$. Further individual analysis of the participants data was not undertaken as this study was focused on validating the accuracy of smart bands rather than the performance of an individual participant.

It can be concluded that the IMU used in this study is the most appropriate substitute for the motion tracking system which is considered gold standard in athlete motion tracking. The accuracy of the Xiaomi smart band can be considered as good as the accuracy of the IMU and therefore can also substitute the motion tracking system in terms to find the number of steps during running.

The grown availability of these small size and weight smart bands allow a wider use for each individual athlete. Nevertheless there are still large differences in accuracy of these devices, with some devices being capable of substituting more expensive laboratory equipment such as IMU's or motion tracking systems. A good smart band therefore allows athletes or hobby sportsmen to relatively accurately track their training progress for every conducted training session.

Acknowledgments: The authors would like to thank all participants who participated in this study.

Conflicts of Interest: The authors declare no conflict of interest.

\section{References}

1. Wang, J.R.; Parameswaran, N. Survey of sports video analysis: Research issues and applications. In Proceedings of the Pan-Sydney Area Workshop on Visual Information Processing, Sydney, Australia, 1 June 2004; pp. 87-90.

2. Terrier, P.; Schutz, Y. Variability of gait patterns during unconstrained walking assessed by satellite positioning (GPS). Eur. J. Appl. Physiol. 2003, 90, 554-561.

3. Wixted, A.J.; Billing, D.C.; James, D.A. Validation of trunk mounted inertial sensors for analysing running biomechanics under field conditions, using synchronously collected foot contact data. Sports Eng. 2010, 12, 207-212.

4. Alonge, F.; Cucco, E.; Ippolito, F.; Pulizzotto, A. The Use of Accelerometers and Gyroscopes to Estimate Hip and Knee Angles on Gait Analysis. Sensors 2014, 14, 8430-8446.

5. Tong, K.; Granat, M.H. A practical gait analysis system using gyroscopes. Med. Eng. Phys. 1999, 21, 87-94.

6. Espinosa, J.L.H.G.; James, D.A. The inertial sensor: A base platform for wider adoption in sport science applications. J. Fit. Res. 2015, 4, 13-20.

7. U.N.O. LTX6. 2017. Available online: http://www.benysports.de/assets/bedienungsanleitung_ltx6_instmanual-2012.12.20.pdf (accessed on 1 December 2017).

8. FitBit. Alta. Available online: https://www.fitbit.com/uk/alta\#features (accessed on 1 December 2017).

9. Samsung. Gear Fit SM-R350. Available online: http://www.samsung.com/uk/wearables/gear-fit-r350/ (accessed on 1 December 2017).

10. Vidonn. X6. Available online: http://en.vidonn.com/ProductX6.html (accessed on 1 December 2017).

11. Xiaomi. MiBand 2. Available online: http://www.mi.com/en/miband2/ (accessed on 1 December 2017).

12. OptiTrack. Prime 13. Available online: http://optitrack.com/products/prime-13/ (accessed on 1 December 2017).

13. OptiTrack. Motive. Available online: http://optitrack.com/products/motive/ (accessed on 1 December 2017).

14. Lai, A.; James, D.A.; Hayes, J.P.; Harvey, E.C. Semi-automatic calibration technique using six inertial frames of reference. In Proceedings of the Microelectronics: Design, Technology, and Packaging, Perth, Australia, 30 March 2004.

15. Stamm, A.; Thiel, D.V. Investigating Forward Velocity and Symmetry in Freestyle Swimming Using Inertial Sensors. Procedia Eng. 2015, 112, 522-527.

(C) 2018 by the authors; Licensee MDPI, Basel, Switzerland. This article is an open access article distributed under the terms and conditions of the Creative Commons Attribution (CC BY) license (http://creativecommons.org/licenses/by/4.0/). 